\title{
Unpredicted Disasters Such as Coronavirus Disease (COVID-19) Highlight the Importance of Teledermatology
}

\author{
S. Morteza Seyed Jafari Robert E. Hunger \\ Department of Dermatology, Inselspital, Bern University Hospital, Bern, Switzerland
}

Dear Editor,

In December 2019, unexplained pneumonia cases were initially reported in China. The pathogen, a novel coronavirus called severe acute respiratory syndrome coronavirus 2 (SARS-CoV-2), termed COVID-19 (coronavirus disease 2019), has rapidly spread all over the world [1]. This time has become a challenging era for dermatologists and dermatologic clinics, since in addition to routine dermatology appointments, they should cope with COVID-19 patients, which often present with cutaneous manifestations of SARS-CoV-2 and/or aggravation of their previous skin diseases such as rosacea, eczema, or atopic dermatitis $[1,2]$. In addition, patients with severe chronic inflammatory skin disorders taking certain immunomodulators have been uncertain about their therapies and appropriate protection. Furthermore, because of frequent hand hygiene and long-term wearing of tertiary protective devices, skin damage was observed more often among healthcare workers [3]. Despite this significant need for dermatologic consultation, many clinics had to be closed, since due to a limited supply of healthcare providers, dermatology residents and attending physicians have been treating COVID-19 patients in many countries [2, 4]. Additionally, because people

karger@karger.com

(c) 2020 S. Karger AG, Basel

www.karger.com/drm

Karger! should stay home during coronavirus outbreaks, many patients with skin problems have to postpone their appointments due to medical risk factors. Furthermore, due to the possible danger of COVID-19 transmission, immediate cessation of nonemergent dermatology visits was proposed [4].

Teledermatology might have the potential to cope with unexpected conditions such as COVID-19 by improving access to dermatological expertise and quality of care while minimizing contacts and the risk of transmission, since the evaluation and diagnosis of skin disorders with accompanying clinical histories can be performed remotely using a number of modalities such as clinical images or live video teleconferencing [5]. Thanks to this important and practical feature, teledermatology can be applied internationally to support colleagues and help patients in countries with more serious conditions as well. Moreover, online consultation and implementation of telemedicine platforms might be a compromise for any dermatology clinic helping them with loans and other forms of financial relief to keep practices running during this crisis [4].

Overall, this unusual situation might be a good opportunity not only for dermatologists to gain more ex- 
perience in treating patients with acute and chronic dermatological problems using such platforms, but also for patients to obtain proper consultation. This might gradually shift patients' preferences towards making greater use of these modern technologies in dermatology and attending telemedicine platforms more regularly, even after the COVID-19 crisis. During this intense time, however, dermatologists should also use their observations and patients' feedback to improve the existing teledermatology platforms by making them quicker, more user-friendly, more reliable, and more efficient.

\section{Key Message}

Teledermatology has the potential to cope with unexpected conditions by improving access to dermatological expertise.

\section{Statement of Ethics}

No ethical approval was needed.

\section{Conflict of Interest Statement}

The authors declare that they have no competing interests.

\section{Funding Sources}

No funding was received.

\section{Author Contributions}

R.E.H. and S.M.S.J. designed the study and structure of the manuscript. S.M.S.J. wrote the paper. R.E.H. and S.M.S.J. revised the paper.

\section{References}

1 Recalcati S. Cutaneous manifestations in COVID-19: a first perspective. J Eur Acad Dermatol Venereol. 2020 May;34(5):e212-3.

2 Zheng Y, Lai W. Dermatology staff participate in fight against Covid-19 in China. J Eur Acad Dermatol Venereol. 2020 May; 34(5):e210-1.

3 Lan J, Song Z, Miao X, Li H, Li Y, Dong L, et al. Skin damage among health care workers managing coronavirus disease-2019. J Am Acad Dermatol. 2020 May;82(5):1215-6.
4 Kwatra SG, Sweren RJ, Grossberg AL. Dermatology practices as vectors for COVID-19 transmission: a call for immediate cessation of nonemergent dermatology visits. J Am Acad Dermatol. 2020 May;82(5):e179-80.

5 Lee JJ, English JC 3rd. Teledermatology: a review and update. Am J Clin Dermatol. 2018 Apr;19(2):253-60. 